\title{
Figure S13
}

FAN
MKWATLLKGTEDPEWQLCPIEGPYRMRKKLERCKLKIDSIQNVLDGKLELGEIELPKVKN

SFDKNRFQNI SEKLHMECKAEM-- - - - HLEKIRFRYNCERVVDLDKHDGIFLIGEFCLYVIENFYIDEDGCICEKECEDELSVIDQA $::^{*} * *$ :

GYPKPVVQITLQDVRRIYKRRHGLMPLGLEVFCTEDDLCSDIYLKFYEPQDRDDLYFYIA NLPHPWRMWKLNNVHEILKRDYQLRPVAIEIFSMD--GCNDLLV--FHKKEREEVFKNLV $*^{*} \quad *^{*}::^{*}:$ * $^{* *}: *^{*}: .:^{*}:^{*} .: *^{*}:: \quad: .:^{*}:::: \quad:$.

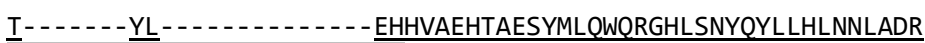
AMNLPRNSMLDTTISGSAKQESNEGGRLFKLMAKSFSKRWQNGEISNFQYLMHLNTLAGR

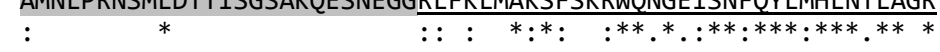

SCNDLSQYPVFPWIIHDYSSSELDLSNPGTFRDLSKPVGALNKERLERLLTRYQ- - - EMP

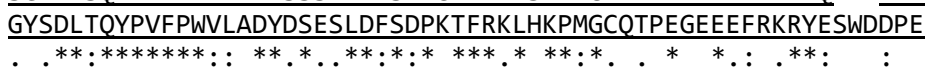

EPKFMYGSHYSSPGYVLFYLVRIAPEY - -MLCLQNGRFDNADRMFNSIAETWKNCLD- - $\underline{G}$ VPKFHYGSHYSSAGIVLFYLIRLPPFSSENQKLQGGQFDHADRLFNSIKDTWLSAAGKGN

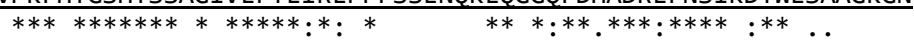

ATDFKELIPEFYGDDVSFLVNSLKLDLGKRQGGQMVDDVELPPWASS-PEDFLQKSKDAL TSDVKELIPEFFYM-PEFLENRFSLDLGEKQSGEKVGDVFLPPWARGSVREFILKHREAL

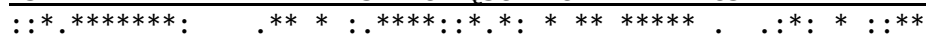

ESNYVSEHLHEWIDLIFGYKQKGSDAVGAHNVFHPLTYEGGVDLNSIQDPDEKVAMLTQI

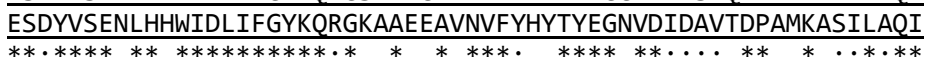

LEFGQTPKQLFVTPHPRRITPKFKSLSQTSSYNASMADSPGEESFEDLTEESKTLAWNNI NHFGQTPKQLFPKAHVKRRTDRKIPLHPLK - - -HSMHLVPHEI - - - - - - -RKCS-SSI $. * * * * * * * * . *^{*}::^{*}$. $* * * * \quad: \quad$. $^{*}$

T-KLQLHEHYKIHKEAVTGITVSRNGSSVFTTSQDSTLKMFSKESKMLQRSISFSNMALS SQIITFHDKV- $::{ }^{*}:: \quad{ }^{*}:$

SCLLLPGDATVITSSWDN- - -NVYFYSIAFGRRQDTLMGHDDA - - - - - VSKICWHDNR NCFLKPRGYTKY-ITWGFPDRSLRFMSY----DQDKLLSTHENLHESNQIQCAGVSHDGR

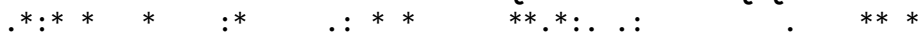

L-YSASWDSTVKVWSGVPAEMPGTKRHHFDLLAELEHDVSVDTISLNAASTLLVSGTKEG IVVTGAEDGLVCVWRVSKDGPRGSRRLRLEK-ALCAHTAKVTCLRVSQPYMMIASGSDDC

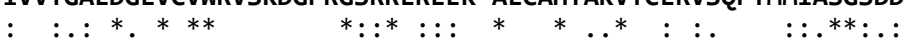

TVNIWDLTTATLMHQIPCHSGIVCDTAFSPDSRHVLSTG - - - - - TDGCLNVIDVQ TVIIWDLSSLSFVRQLPDFPVPISAIYIN-DLTGEIVTAAGTVLAVWSINGDCLAVANTS

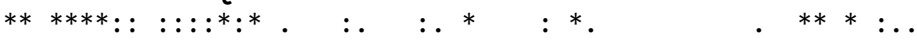

IGMLISSMTSDEPQRCFVW-DGNSVLSGSQSGELLVWDLLGAKISERIQ- - - - - QL-PSDSVLSVTGSTSSDWLETSWYVTGHQSGAVKVWRMIHCTDPVSAESKTSSSNRTGG 940

$$
.^{*}: *^{*} . *^{*}: \text {. }::^{*} * * *: *^{*}:: \text { : : }
$$

